\title{
SPORTS NUTRITION: WHAT THE FUTURE MAY BRING
}

\author{
Douglas S. Kalman ${ }^{1}$ and Bill Campbell ${ }^{2}$ \\ Miami Research Associates, Miami, FL ${ }^{1}$; Exercise \& Sport Nutrition Lab, Baylor University, \\ Waco, TX'2. Address correspondence to DKalman@miamiresearch.com. \\ Received March 26, 2004/Accepted May 18, 2004/Published (online)
}

\section{ABSTRACT}

The field of sports nutrition is a dynamic one. Core competencies in exercise physiology, psychology, integrated metabolism and biochemistry are the initial parameters for a successful career in sports nutrition. In addition to the academic fundamentals, it is imperative that the sports nutritionist understand the sport in which our client participates. This sport specific understanding should manifest itself in fuel utilization, mechanics of movement, as well as psychological processes that motivate the participant to perform optimally. Sports nutrition as a field has grown substantially over the past 50 years, from glycogen loading to today's scientifically validated ergogenic aids. The last ten years has seen the largest advancement of sports nutrition, with the following areas driving much of the research: the effects of exercise on protein utilization, meal timing to maximize the anabolic response, the potential for ribose to benefit those engaged in high-energy repetitive sports, and creatine and its uses within athletics and medicine. The future of sports nutrition will dictate that we 1) collectively strive for a higher standard of care and education for counseling athletes and 2) integrate different disciplines. We are in an era of unprecedented growth and the new knowledge is constantly evolving. The International Society of Sports Nutrition (ISSN) will contribute to this exciting field in many ways, and we ask for your contribution by sharing your passion, stories, research, and life experiences with us. Sports Nutrition Review Journal. 1(1):61-66, 2004.

\section{Key Words: sport nutrition, ergogenic aids}

\section{INTRODUCTION}

The field of nutrition is a dynamic one. Athletes often ask their trainers, physiologists, coaches, doctors, and dietitians for guidance related to what to eat and which supplements to use. Registered Dietitians have choices to work within clinical dietetics, nutrition support, research, outpatient or private counseling, consulting to the food industry, consulting to the supplement industry, direct food or supplement industry employment, in product development and many other economical beneficial areas. Exercise physiologists can work in cardiac rehabilitation settings, private health clubs, academic institutions, research organizations, for various private companies (corporate wellness, exercise equipment design, etc.) and other related positions. Often the weekend athlete and the professional athlete will seek nutrition advice from both the dietitian and exercise physiologist. This advice is Sports Nutrition.

Sports Nutritionists may work with individuals, teams, and professional organizations or due to economic influences and opportunity have to split their places of employment (i.e., hospital and private consulting). In our field, the understanding of exercise physiology, 
psychology, integrated metabolism and biochemistry along with ethics are intrinsic ingredients to success. ${ }^{1,2,3}$ Within sports, of any sort, there is always a prevailing notion that the athlete strives to get better or be "bigger, faster and stronger". However, whether the athlete is one that is involved in competitive sports, competes with him or herself, or is a "weekend warrior", our charge is to stay ahead of their curve by staying abreast of the latest relevant findings and then applying these findings. If you take into consideration that our friends, family, patients, clients, magazine editors and others are always asking questions about nutrition and couple these questions with those received by our athletes, and it becomes obvious that the motivation to stay current with knowledge both real and theoretical is warranted.

Sports Nutrition is not a licensed profession (yet). Many organizations (i.e., The International Fitness Professional Association, International Sports Sciences Association, etc.) supply certification examinations for sports nutrition. However, none of these organizations carry the clout of the American Dietetic Association, the American College of Sports Medicine or the National Strength and Conditioning Association. Additionally, none of these organizations are able to obtain recognition for their certifications by state governments or the Department of Education. One member of our organization was stated (paraphrasing) that to be a sports nutritionist, all one had to do is to have taken one or two classes in sports nutrition (which most universities do not offer) and that additional coursework in exercise physiology was not needed. Thus, as long as you took your basic American Dietetic Association mandated coursework and a course in sports nutrition, you were capable of working with any athlete (albeit, amateur or professional). The exercise physiology undergraduate training in nutrition is limited, though through elective courses this can be strengthened (also if the student has a double major or a minor in nutrition). Let's be clear, times have changed and we are involved in enabling change. This change includes the advancement of our profession, sports nutrition.

Sports nutrition is a complex field, and a good sports nutritionist has core competencies in nutrient metabolism (biochemistry and metabolism), exercise physiology, and psychology. Athletes today are demanding that we understand their sport. The traditional learning model has taught basics of anaerobic and aerobic metabolism, but not much emphasis on sport specific energy expenditure, nor the influence of post-exercise oxygen consumption (EPOC). Thus, advanced learning beyond that of a baccalaureate degree should be pursued. The need for advanced education whether it is in the form of continuing education, a legitimate advanced degree certification (perhaps analogous to a Certified Nutrition Support Dietitian or the Exercise Physiologist-Certified) is evident.

Sports nutrition is often considered within the field to have divergent thought leaders. We know that since the science is evolving and that not all keep up-to-date with the latest publications (journals or texts) that not all of us are on the same advising page. Perhaps, controversy is good. If we all were of the same opinion, than what would be the motivation for progress and the pursuit of new knowledge? Take for example, the heated arguments that occur just over the protein needs of athletes as compared to sedentary folk as well as anaerobic versus aerobic athletes. This in and of itself drives the sales of many books and magazines. However, one needs to be cognizant that athletes often view food as one means of obtaining their goals, while ergogenic aids at times are believed to be the missing link which will propel the athlete's performance over the competition. In this situation, knowledge about the safety and efficacy of ergogenic aids is paramount in truly helping your clients. 
If we examine basic sports nutrition guidelines over the past fifty years, it becomes apparent the biggest breakthrough was the discovery of how to glycogen load, refinement of the means of glycogen loading (from the days of depletion followed by super-compensation to tapering exercise duration while concomitantly increasing the diet to almost exclusively carbohydrate), followed by nitrogen balance studies demonstrating a slight increase in needs for athletes as compared to the sedentary and the evolution of creatine monohydrate as an ergogenic aid. ${ }^{4,5}$ However, the last ten years may be the most exciting in the advancement of sports nutrition.

The following areas of nutrition are where the most growth is occurring: evaluating the effects of exercise on protein utilization, thus the overall protein needs, meal timing to maximize the anabolic response, the true "essentiality" of essential amino acids, the potential for ribose to benefit those engaged in high-energy repetitive sports (i.e., football lineman), and creatine and its uses within athletics and medicine. It is up to us and other academic thought leaders to help grow the biological and metabolic understanding of the interaction of foods, nutrients, nutrient supplementation, exercise and the recovery from said exercise as well as the actual performance to the next level.

\section{PROTEIN}

A brief review of protein needs of athletes reveals that the current recommended intakes of protein for strength and endurance athletes are 1.6 to $1.7 \mathrm{~g} / \mathrm{kg}$ and 1.2 to $1.4 \mathrm{~g} / \mathrm{kg}$ per day, respectively. ${ }^{6}$ Presently, most athletes consume an adequate amount of protein in their diet. However, depending upon overall caloric needs and training intensities, the reality of obtaining all nutrition from foods alone will dictate the needs for protein supplementation. The timing and nutritional content of the post-exercise meal, although often overlooked, are known to have synergistic effects on protein accretion after exercise. New evidence suggests that individuals engaging in strenuous activity consume a meal rich in amino acids and carbohydrate soon after the exercise bout or training session. In addition to this, preworkout feedings have been demonstrated to show a greater net effect on skeletal muscle protein synthesis over and above that of postexercise feedings.

\section{NUTRIENT TIMING}

Meal timing and type is important for the athlete and their nutritionist to be aware of. A recent study found that performance of moderate- to high-intensity exercise lasting 35$40 \mathrm{~min}$ is improved by consuming a moderately-high carbohydrate, low fat, low protein meal 3-hr before exercise compared to a similar meal consumed $6 \mathrm{hr}$ prior to exercise. ${ }^{7}$ Thus, athletes should not skip meals before competition or training sessions. However, one should note that while research used exercise duration of about 40 minutes, some of our athletes exercise or compete for hours at a time. In this case, meal timing, snacking, and appropriate liquid supplementation during exercise becomes of paramount importance.

Meal timing has also been shown to have an effect on muscle protein synthesis (MPS). Affecting MPS may allow the downstream creation of new muscle mass (lean body mass). Recent data indicate that consuming a small meal of mixed macronutrient composition (or perhaps even a very small quantity of a few indispensable/essential amino acids) immediately before or following strength exercise bouts can alter significantly net protein balance, resulting in greater gains in both muscle mass and strength than observed with training alone. With aerobic exercise, some evidence suggests immediate post-exercise (but perhaps not pre-exercise) supplementation is also beneficial. Second, protein type may also be important owing to variable speeds of absorption and availability, differences in amino acid and peptide profiles, unique 
hormonal response, or positive effects on antioxidant defense. ${ }^{8}$ In addition to athletes, many others who desire to regain, maintain, or enhance muscle mass or function, including those with muscle-wasting diseases, astronauts, and all of us as we age, need to ensure that nutrient availability is sufficient during the apparently critical anabolic window of time associated with exercise training sessions. The additional data compiled from the cumulative studies examining the effects of low dose essential amino acids with or without carbohydrate (sucrose) indicate that the prudent use of supplemental protein may be the easiest way to augment the MPS response postexercise. ${ }^{9,10,11}$ Since post-exercise carbohydrate intake alone does not affect MPS, but essential amino acids do, there is much more to learn about post-exercise nutrition. Future studies are needed to fine-tune these recommendations.

\section{IS A SUGAR A SUGAR?}

Recent popular press literature has been noted for promoting the pentose, ribose as a dietary supplement. In fact, many patents and patentpending filings exist for the uses of ribose in athletics and medicine. Ribose is a carbohydrate, or sugar, used by all living cells and is an essential component in our body's energy production. The company who holds the ribose patents claims the following on their website: "Ribose is essential in helping the body restore its cellular energy level. The cells use ribose to convert nutrients in ATP, so it makes sense that more ribose means more ATP production. Research shows that ribose increases ATP production in both fast-twitch and slow-twitch muscles by 3.4 to 4.3 times. The catch is, the body only has so much ribose at any one time, and there's no food source that increases the body's ribose level. The good news is that supplemental ribose does just that. Weight lifters; sprinters; football, basketball and soccer players; triathletes; cyclists and other athletes requiring sudden high-energy bursts may benefit from supplemental ribose.
Energy recovery in muscle cells during and after strenuous high-intensity exercise is enhanced with ribose supplementation. Ribose increases both de novo (new) synthesis and salvage of nucleotides in heart and skeletal muscle. Research conducted by Bioenergy and others suggests that even low doses of ribose will have an effect on energy recovery following high-intensity exercise. Maintenance doses as low as two grams per day will increase recovery of lost nucleotides by increasing de novo synthesis and salvage of nucleotides that are in danger of being lost due to strenuous activity. Further, there appears to be a functional benefit of ribose loading prior to a high-intensity event. Bioenergy continues to investigate this effect."12

Published research in peer-reviewed journals does not support the hyperbole behind the purported use of ribose. It should be noted that oral D-ribose supplementation has been reported to increase adenine nucleotide synthesis (TAN) and exercise capacity in certain clinical populations. Increasing TAN, may cause the downstream increase in ATP production during times of stress. Analysis of uses in anaerobic exercise and during sprint cycling from recent published studies does not support the use of ribose for athletes. ${ }^{13,14}$ More research is needed to determine if there is an applicability of ribose within athletic protocols.

\section{IS HMB MORE THAN A HYPED LEUCINE DERIVATIVE?}

Beta-hydroxy-beta methylbutyrate (HMB) is a purported anti-catabolic agent. HMB is a downstream metabolite of the branched chain amino acid leucine. A recent meta-analysis concluded that HMB along with creatine are two dietary supplements that have substantial evidence indicating a positive effect on lean mass accretion when used with resistance exercise. ${ }^{15}$ However, the meta-analysis and most of the studies published on HMB have been conducted by the patent holder(s) and/or 
staff of the company who distributes HMB (MTI Biotech). Independent studies have found either no effect for enhancing lean body mass or only minor protective effects against stress induced muscle damage (via mitigation of creatine phosphokinase and/or lactate dehydrogenase: CPK, LDH). More research is needed in order to determine if there is a true benefit for aerobic (no current research exists for examining the effect of HMB on time to exhaustion or respiratory quotient during exercise) or for anaerobic athletes as an anticatabolic agent.

\section{CREATINE, STILL EFFICACIOUS AFTER 10 YEARS OF POPULARITY}

Creatine has probably made the biggest impact on the sports nutrition landscape. To date, there are over 500 studies on this ergogenic aid. To date, the most promising strategies to augment gains in muscle size and strength appear to be consumption of protein-carbohydrate calories before and after resistance exercise, and creatine supplementation. ${ }^{16}$ Short-term creatine supplementation (e.g. 20 g/day for 5-7 days) has typically been reported to increase total creatine content by $10-30 \%$ and phosphocreatine stores by $10-40 \%$. Short-term creatine supplementation has been reported to improve maximal power/strength (5-15\%), work performed during sets of maximal effort muscle contractions (5-15\%), single-effort sprint performance (1-5\%), and work performed during repetitive sprint performance (5-15\%). No study to date has found an ergolytic effect of creatine. Creatine supplementation during training has been reported to promote significantly greater gains in strength, fat free mass, and performance primarily of high intensity exercise tasks. ${ }^{17}$ Not all of the studies examining athletic uses demonstrate an ergogenic effect; approximately $30 \%$ do not support the agent, although some report non-significant positive effects or influence of creatine monohydrate. There have also been some concerns associated with anecdotal reports in relation to creatine safety and injury data with athletes (muscle cramping, dehydration, and gastrointestinal distress). The information that has been scientifically validated concerning these issues calls into question the claims raised by these anecdotal claims. ${ }^{18,19}$ From a clinical perspective, research has demonstrated a number of potentially beneficial uses of creatine in heart patients, patients suffering orthopedic injury, muscle wasting diseases such as AIDS, and patients with various neuromuscular diseases. Future research will determine what dose may be best for athletic uses. Also, as food science evolves, stabilized liquid forms of creatine for ease of use are expected to be developed.

\section{HELP THE ISSN DEVELOP THE FUTURE OF SPORTS NUTRITION}

The future of sports nutrition will dictate that we collectively will have to have a higher standard of care and education for counseling athletes, whether individually or in groups. The integration of many different disciplines

(physiology, metabolism, psychology, etc.) will become a minimum mandatory set of disciplines for any aspiring sports nutritionist. A standardized certification is also expected to be available to Registered Dietitians and Ph.D.'s (in related areas) within the next twoyears, this certification will help the public to decipher the true sports nutritionist from the one who has read many popular press articles and has not been exposed to the same educational rigor as others. The International Society of Sports Nutrition (ISSN) is currently working on this certification. We are in an era of unprecedented growth and the new knowledge is evolving. This exciting time that we live in can be driven further by all of us sharing our passion to help others while being open minded to the discoveries that await us. Please share your stories, research and life experiences with us. 


\section{REFERENCES}

1. James E. Loehr (editor). The New Toughness Training for Sports: Mental, Emotional, and Physical Conditioning from One of the World's Premier Sports Psychologists. James E. Leohr, Chris Evert, Dan Jansen, Plume; Reprint edition (November 1995)

2. Robert N. Singer, Heather Ann Hausenblas, and Christopher Janelle (Editors). Handbook of Sport Psychology. Publisher: John Wiley \& Sons; 2nd edition (January 15, 2001).

3. Hargreaves M (Editor). Exercise Metabolism 1995 Human Kinetics, Champaign, IL.

4. Costill DL. Inside Running: Basics of Sports Physiology. 1986 Brown and Benchmark, Dubuque, IA.

5. Wolinsky I. (editor). Nutrition in Exercise and Sport. 1998. CRC Press, Boca Raton, Fl.

6. Fielding RA, Parkington J. What are the dietary protein requirements of physically active individuals? New evidence on the effects of exercise on protein utilization during post-exercise recovery. Nutr Clin Care. 2002;5(4):191-6.

7. Maffucci DM, McMurray RG. Towards optimizing the timing of the pre-exercise meal. Int J Sport Nutr Exerc Metab. 2000;10(2):103-13.

8. Lemon PW, Berardi JM, Noreen EE. The role of protein and amino acid supplements in the athlete's diet: does type or timing of ingestion matter? Curr Sports Med Rep. 2002;1(4):214-21.

9. Miller SL, Tipton KD, Chinkes DL, Wolf SE, Wolfe RR. Independent and combined effects of amino acids and glucose after resistance exercise. Med Sci Sports Exerc. 2003;35(3):449-55.

10. Wolfe RR. Regulation of muscle protein by amino acids. J Nutr. 2002;132(10):3219S-24S.

11. Borsheim E, Tipton KD, Wolf SE, Wolfe RR. Essential amino acids and muscle protein recovery from resistance exercise. Am J Physiol Endocrinol Metab. 2002;283(4):E648-57.

12. www.ribose.com Accessed July 19, 2003

13. Kreider RB, Melton C, Greenwood M, Rasmussen C, Lundberg J, Earnest C, Almada A. Effects of oral D-ribose supplementation on anaerobic capacity and selected metabolic markers in healthy males. Int J Sport Nutr Exerc Metab. 2003;13(1):76-86.

14. Berardi JM, Ziegenfuss TN. Effects of ribose supplementation on repeated sprint performance in men. J Strength Cond Res. 2003;17(1):47-52.

15. Nissen S, Sharp R. Effect of dietary supplements on lean mass and strength gains with resistance exercise: a meta-analysis. Scand J Med Sci Sports. 2003;13(4):272.

16. Volek JS. Strength nutrition. Curr Sports Med Rep. 2003;2(4):189-93.

17. Kreider RB. Effects of creatine supplementation on performance and training adaptations. Mol Cell Biochem. 2003;244(1-2):8994.18

18. Kreider R.B, Melton C, Rasmussen C, Greenwood M, Lancaster S, Cantler E, Milnor P, Almada A. Long-term creatine supplementation does not significantly affect clinical markers of health in athletes. Mol Cell Biochem. 2003;244:95-104

19. Greenwood M, Kreider RB, Greenwood L, Byers A. Cramping and injury incidence in collegiate football players are reduced by creatine supplementation. J Athl Train. 2003 Sep;38(3):216-219 